\title{
Bayesian multi-tensor factorization
}

\author{
Suleiman A. Khan ${ }^{1,2}$ - Eemeli Leppäaho' ${ }^{1}$. \\ Samuel Kaski ${ }^{1}$
}

Received: 31 March 2015 / Accepted: 7 March 2016 / Published online: 10 June 2016

(C) The Author(s) 2016

\begin{abstract}
We introduce Bayesian multi-tensor factorization, a model that is the first Bayesian formulation for joint factorization of multiple matrices and tensors. The research problem generalizes the joint matrix-tensor factorization problem to arbitrary sets of tensors of any depth, including matrices, can be interpreted as unsupervised multi-view learning from multiple data tensors, and can be generalized to relax the usual trilinear tensor factorization assumptions. The result is a factorization of the set of tensors into factors shared by any subsets of the tensors, and factors private to individual tensors. We demonstrate the performance against existing baselines in multiple tensor factorization tasks in structural toxicogenomics and functional neuroimaging.
\end{abstract}

Keywords Bayesian factorization - CANDECOMP/PARAFAC · Coupled matrix tensor factorization $\cdot$ Factor analysis $\cdot$ Tensor factorization

\section{Introduction}

Matrix and tensor factorization methods have been studied for data analysis and machine learning for decades. These methods decompose a single data set into a low-dimensional rep-

Editor: Kristian Kersting.

Suleiman A. Khan

khan.suleiman@gmail.com; suleiman.khan@helsinki.fi

$凶 \quad$ Samuel Kaski

samuel.kaski@aalto.fi

Eemeli Leppäaho

eemeli.leppaaho@aalto.fi

1 Department of Computer Science, Aalto University, P.O. Box 15400, 00076 Aalto, Finland

2 Institute of Molecular Medicine Finland FIMM, University of Helsinki, Helsinki, Finland 
resentation of factors that explain the variation in it. With linked data sets becoming increasingly common, joint factorization of multiple data sources is now gaining significant attention.

Joint factorization of multiple matrices integrates information from multiple coupled data sets. It decomposes them into underlying latent components or factors, taking advantage of the common structure between all of them. For the simplest case of two paired matrices, canonical correlation analysis finds latent variables that capture the shared variation explaining them (Bach and Jordan 2005; Hardoon et al. 2004; Hotelling 1936). While canonical correlation analysis searches for common patterns between two data matrices, its straightforward extensions have limited applicability in multiple coupled matrices. Recently, a multi-view method called group factor analysis (GFA; Klami et al. 2015; Virtanen et al. 2012), has been presented for decomposing multiple paired matrices. GFA decomposes multiple coupled matrices identifying both the co-variation patterns shared between some of the data sets, as well as those specific to each.

Tensor factorizations have also been considered as a means of analyzing multiple matrices by coupling them together as slabs of a tensor. These factorizations are more general and are able to take advantage of the natural tensor structure of the data. A host of lowdimensional tensor factorization methods have been proposed earlier (see Kolda and Bader 2009, forareview). The most well-known are the CANDECOMP/PARAFAC (CP; Carroll and Chang 1970; Harshman 1970) and the Tucker family of models (Kiers 1991; Tucker 1966). CP assumes a trilinear structure in the data and is easier to interpret, while the Tucker family defines more generic models for complex interactions.

However, neither the tensor factorization nor the joint matrix factorization is able to factorize mixed and partially linked data sets. Recently, fusion of partially coupled data sets has been discussed, for example to predict the values in a tensor with side information from a matrix, or vice versa. For example, Acar et al. (2013b) used metabolomics data of fluorescence emission $\times$ excitation measurements and NMR recordings of several human blood samples to form a coupled tensor and a matrix, to demonstrate that joint factorization outperforms individual factorization. The concept of such multi-block decompositions was originally introduced by Smilde et al. (2000), and proposed by Harshman and Lundy (1994), though the recent formulation by Acar et al. $(2011,2013 \mathrm{~b})$ has brought coupled matrix tensor factorizations to practical use.

We call this general research problem multi-tensor factorization (MTF) and present the first Bayesian formulation for an extension of joint matrix-tensor factorization. We also present the first generalized formulation of multi-tensor factorization to arbitrary tensors and introduce a relaxed low-dimensional decomposition that allows the tensor to factorize flexibly. Our model decomposes multiple co-occurring matrices and tensors into a set of underlying factors that can be shared between any subset of them, with an intrinsic solution for automatic model selection. Finally, we demonstrate the use of the method in novel coupled matrix-tensor factorization applications, including structural toxicogenomics and stimulusresponse prediction in neuroimaging.

The rest of the paper is structured as follows: In Sect. 2, we start by formulating the special case of a single matrix and single tensor factorization, inferring components that are shared between both of them, or are specific to either one. In Sect. 3, we present our Bayesian model that extends to multiple paired tensors and matrices. In Sect. 4 we introduce an extension of our new Bayesian solution of Sect. 3 that automatically tunes the decomposition structure for the data. We propose a generic formulation in Sect. 5, and discuss special cases and related works in Sect. 6. We validate the performance of our models in various technical experiments in Sect. 7, and demonstrate their applicability in a neuroimaging stimulus-response 
relationship study and in a novel structural toxicogenomics setting in Sect. 8. We conclude with discussion in Sect. 9.

Notations: We denote a tensor as $\mathcal{X}$, a matrix as $\mathbf{X}$, a vector as $\mathbf{x}$ and a scalar as $x$. As presented in Kolda and Bader (2009), the Mode-1 product $\times{ }_{1}$ between a tensor $\mathcal{A} \in \mathbb{R}^{K \times D \times L}$ and a matrix $\mathbf{B} \in \mathbb{R}^{N \times K}$ is the projected tensor $\left(\mathcal{A} \times{ }_{1} \mathbf{B}\right) \in \mathbb{R}^{N \times D \times L}$, that reshapes the first mode of the tensor. A Mode- 2 product $\times_{2}$ similarly reshapes the 2 nd mode. The outer product of two vectors is denoted by $\circ$ and the element-wise product by $*$. The order of a tensor is the total number of axes, modes or ways in the tensors, while tensor rank is the smallest number of rank-1 component tensors that generate it; an $N$ th order rank one tensor $\mathcal{X}$ can be presented as $\mathbf{w}_{1} \circ \cdots \circ \mathbf{w}_{N}$. For notational simplicity we present the models for third order tensors only, including matrices, for which the dimension of the third mode is one, i.e. $\mathcal{X} \in \mathbb{R}^{N \times K \times 1}$.

\section{Matrix tensor factorization}

We formulate the joint matrix tensor factorization problem as the identification of a combined low-dimensional representation of the matrix $\mathcal{X}^{(1)} \in \mathbb{R}^{N \times D_{1} \times 1}$ and the tensor $\mathcal{X}^{(2)} \in \mathbb{R}^{N \times D_{2} \times L}$ such that each underlying factor is either shared by both the matrix and the tensor, or is private to one of them. The matrices and tensors can jointly be referred to as different views of the data, analogously to the terminology used in multi-view learning. The shared factors represent variation that is common in both the views, while specific components capture the view-specific variation.

The joint factorization can be defined, with a common set of low-dimensional latent variables $\mathbf{Z} \in \mathbb{R}^{N \times K}$, loading matrix $\mathcal{W}^{(1)} \in \mathbb{R}^{K \times D_{1} \times 1}$ and loading tensor $\mathcal{W}^{(2)} \in \mathbb{R}^{K \times D_{2} \times L}$, for each view $t$ as

$$
\mathcal{X}^{(t)}=\mathcal{W}^{(t)} \times{ }_{1} \mathbf{Z}+\boldsymbol{\epsilon}^{(t)}
$$

where $\boldsymbol{\epsilon}^{(1)} \in \mathbb{R}^{N \times D_{1} \times 1}$ is a matrix representing noise, while $\boldsymbol{\epsilon}^{(2)} \in \mathbb{R}^{N \times D_{2} \times L}$ is a noise tensor. The factorization of the tensor $\mathcal{X}^{(2)}$ into $\mathcal{W}^{(2)} \times_{1} \mathbf{Z}$, where $\mathcal{W}^{(2)}$ is unconstrained, is equivalent to Tucker-1 factorization (Kiers 1991), which is analogous to matrix factorization of a matricized tensor. This factorization still has a huge number of parameters, and the loadings $\mathcal{W}^{(2)}$ can be further factorized to model tensorial interactions. A popular choice is the CP-type formulation which captures trilinear relationships (Fig. 1) and has the advantage of being easier to interpret. CP is equivalent to a sum of rank-1 tensors where each rank-1 tensor is the outer product of vector loadings in all modes. This rank-1 component decomposition of $\mathrm{CP}$ makes it the choice in most studies. For other useful properties of $\mathrm{CP}$, such as the so-called intrinsic axis property from parallel proportional profiles, see Cattell (1944) and Harshman (1970).
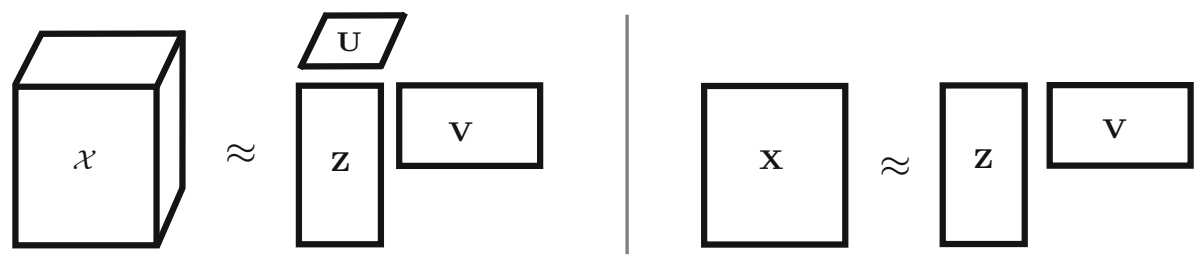

Fig. $1 \mathrm{CP}$ factorization (left) is analogous to matrix factorization (right); each matrix slab of the tensor is just scaled by the corresponding value of each component in an additional matrix $\mathbf{U}$ 


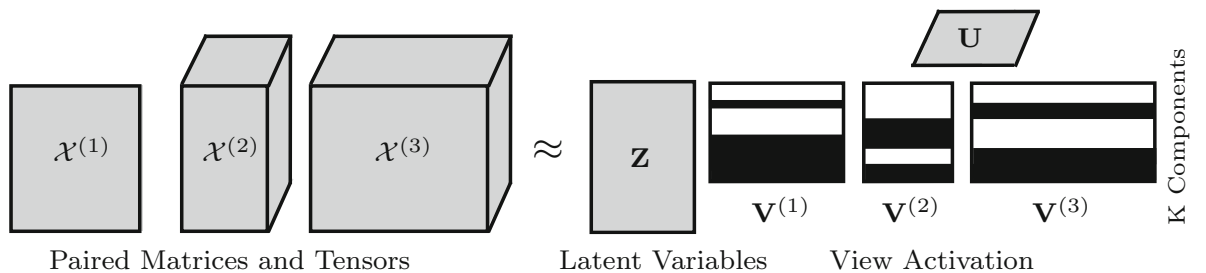

Fig. 2 Multi-tensor factorization projects a set of coupled matrices and tensors into a joint low-dimensional space. In this case, one matrix $\mathcal{X}^{1}$ and two tensors $\mathcal{X}^{2}$ and $\mathcal{X}^{3}$ are decomposed into a common set of latent variables $\mathbf{Z}$ and view-specific projection matrices $\mathbf{V}^{(1,2,3)}$, with the two tensor decompositions having an additional joint set of latent variables $\mathbf{U}$ for the $3 \mathrm{rd}$ mode. The $\mathbf{V}$ 's control the activity of each component in each view, with black representing a component active in a view, white being switched off. A component active (black) in two or more views captures common patterns of variation

Assuming a CP-type decomposition, the loading tensor $\mathcal{W}^{(2)}$ is factorized into a tensor product of two latent variable matrices $\mathbf{V}^{(2)} \in \mathbb{R}^{D_{2} \times K}$ and $\mathbf{U}^{(2)} \in \mathbb{R}^{L \times K}$. Noting that matrix factorization is a special case of $\mathrm{CP}$, both of them can be expressed in the same way, reformulating the joint decomposition as

$$
\mathcal{X}^{(t)}=\sum_{k=1}^{K} \mathbf{z}_{k} \circ \mathbf{v}_{k}^{(t)} \circ \mathbf{u}_{k}^{(t)}+\boldsymbol{\epsilon}^{(t)},
$$

where the factorization of matrix $\mathcal{X}^{(1)}$ corresponds to Eq. 2 with $\mathbf{U}^{(1)}$ as $\mathbf{1}^{1 \times K}$.

A key property of our joint factorization is that each factor can be shared by both the matrix and the tensor, or be specific to either one of them. This can be achieved by imposing a group-sparse prior on the loading matrix $\mathbf{V}^{(t)}$ of each view, similar to that in Virtanen et al. (2012). The group-sparse prior controls which of the $k$ latent variables are active (i.e., non-zero) in each view. A component active in both views is said to be shared between them, while a component active in only one captures variation specific to that particular view. This formulation allows the matrix and tensor to be decomposed comprehensively, while simultaneously identifying the common and specific patterns.

\section{Multi-tensor factorization}

We present the first Bayesian treatment of matrix tensor factorization while simultaneously extending it to collections of multi-view matrices and tensors, coupled by having a common set of samples. The proposed factorization framework is very general, and could as well go by the names collective or collaborative matrix-tensor factorization, or other similar names. Acknowledging that matrices are two-dimensional tensors, we formulate the model family in a simpler but equivalent way as multi-tensor factorization.

Figure 2 illustrates the MTF problem for one matrix and two tensors. The samples couple one mode across the collection, and two modes for the tensors. The task now is to perform a joint decomposition of the matrix and the tensors, distinguishing also between the shared and private components. Assuming an underlying CP decomposition for the tensor as in Eq. 2, we perform an unsupervised joint factor analysis and CP-type decomposition of the matrices and tensors, respectively. The joint decomposition is characterized by (i) $\mathbf{Z}$, a common set of latent variables in all the views (matrices and tensors), (ii) $\mathbf{U}$, the latent variables that 
model the third mode common to the two tensor views $\left(\mathcal{X}^{(2)}, \mathcal{X}^{(3)}\right)$ only and (iii) $\mathbf{V}^{(t)}$, the view-specific loadings that control which patterns from $\mathbf{Z}$ and $\mathbf{U}$ are reflected in each view.

This factorization can be seen as a joint FA-CP decomposition where variation patterns can be shared between matrices and tensors, or be specific to each. It is motivated by its two main characteristics. First, the decomposition of all the matrices and tensors is coupled with the latent variables $\mathbf{Z}$ that capture the common response patterns, enabling the model to capture dependencies between all the views for learning a better factorization. Second, the decomposition allows each factor to be active in any combination of the matrices and tensors. This gives the formulation the ability to capture the underlying dependencies between all or some of the data views, as well as to segregate them from the variation that is specific to just one view, often interpretable as (structured) noise. The dependencies between the views are learned in a fully data-driven fashion, automatically inferring the nature of each type of dependency.

Formally, we define multi-tensor factorization for the collection of $t=(1, \ldots, T)$ coupled tensors and matrices, $\mathcal{X}^{(1)}, \mathcal{X}^{(2)}, \ldots, \mathcal{X}^{(T)} \in \mathbb{R}^{N \times D_{t} \times L_{t}}$, each referred to as a data view. The central assumption, which will be relaxed later in Sect. 5, is that all the views are coupled in one common mode. Assuming normal distributions and conjugate priors, the generative model underlying the joint matrix-tensor factorization can be expressed as

$$
\begin{aligned}
& x_{n, d, l}^{(t)} \sim \mathcal{N}\left(\mathbf{z}_{n}^{\top}\left(\mathbf{v}_{d}^{(t)} * \mathbf{u}_{l}^{(t)}\right), \tau_{t}^{-1}\right) \\
& z_{n, k} \sim \mathcal{N}(0,1) \\
& v_{d, k}^{(t)} \sim h_{t, k} \mathcal{N}\left(0,\left(\alpha_{d, k}^{(t)}\right)^{-1}\right)+\left(1-h_{t, k}\right) \delta_{0} \\
& u_{l, k}^{(t)} \sim \begin{cases}1, & \text { if } L_{t}=1, \\
\mathcal{N}(0,1), & \text { otherwise. }\end{cases} \\
& h_{t, k} \sim \operatorname{Bernoulli}\left(\pi_{k}\right) \\
& \pi_{k} \sim \operatorname{Beta}\left(a^{\pi}, b^{\pi}\right) \\
& \alpha_{d, k}^{(t)} \sim \operatorname{Gamma}\left(a^{\alpha}, b^{\alpha}\right) \\
& \tau_{t} \sim \operatorname{Gamma}\left(a^{\tau}, b^{\tau}\right),
\end{aligned}
$$

where further constraints can be set depending on the data structure (e.g. $\mathbf{U}^{(2)}=\mathbf{U}^{(3)}$ in the case of Fig. 2). The generative model differs for true tensors $\left(L_{t}>1\right)$ and matrices $\left(L_{t}=1\right)$ only in terms of the parameter $\mathbf{U}^{(t)}$. This formulation is similar to Eq. 2, with the separate noise precision $\tau_{t}$ for each view. For a matrix view, $\tau_{t}$ samples the noise matrix, and a tensor for a tensor view. The Gaussian latent variables $\mathbf{Z}$ are shared across all views, while $\mathbf{U}$ depends on the matrix views only indirectly (via $\mathbf{Z}$ ).

The binary variable $h_{t, k}$ controls which components are active in each view, by switching the $\mathbf{v}_{:, k}^{(t)}$ on or off. This is achieved via the spike and slab prior which samples from a two-part distribution (Mitchell and Beauchamp 1988). We center the spike at zero $\left(\delta_{0}\right)$, allowing the components to be shut down, while the slab is sampled from an element-wise formulation of the ARD prior (Neal 1996), parameterized by $\alpha_{d, k}^{(t)}$, enabling active components to have feature-level sparsity. This way the $h_{t, k}$ effectively govern the sharing of components across all the $T$ views, irrespective of whether they are matrices or tensors. The view-specific loading matrices $\mathbf{V}^{(t)}$ capture active patterns in each data view, while containing zeros for all inactive components, as illustrated with the white and black patterns in Fig. 2. 
The learning of $h_{t, k}$ automatically, in a data-driven way, gives the algorithm the power to distinguish between components that are shared between the matrices and the tensors, from those specific to only one of them. This is achieved in an unbiased fashion by placing an uninformative beta-Bernoulli prior on $h_{t, k}$ (default parameters being $a^{\pi}=b^{\pi}=1$ ). The formulation also allows the model to learn the total cardinality of each dataset, as well as of all the data sets combined. This is accomplished by setting $K$ to a large enough value that for a few $k, h_{t, k}$ goes to zero in all $t$ views. Such components will be referred to as empty components, and the presence of empty components indicates that $K$ was large enough to model the data. The effective cardinality of the data set collection is then $K$ minus the number of empty components. Inference of the model posterior can be done with Gibbs sampling and the implementation is publicly available. ${ }^{1}$ The used conjugate priors allow rather straightforward sampling equations, which are omitted here. For the discrete spike and slab prior of $\mathbf{H}$, the sampling was done in a similar fashion as in Klami et al. (2013) for Bayesian canonical correlation analysis. The Gibbs sampling scheme for MTF contains inverting a $K \times K$ covariance matrix, resulting in $\mathcal{O}\left(K^{3}\right)$ complexity, which in general makes the model's runtime practical for $K$ in the order of hundreds or less.

For MTF, the uniqueness of the maximum likelihood estimate, and hence asymptotically of the Bayesian solution $(N \rightarrow \infty)$, follows from the uniqueness proofs of Kruskal (1977), Kolda and Bader (2009), Sørensen and Lathauwer (2015), assuming identifiable structure in the matrices. For full posterior inference with a limited sample size, however, uniqueness is still an open research problem.

\section{Relaxed multi-tensor factorization}

The trilinear CP structure places a strong assumption on the factorization of a tensor, namely that the projection weights in the second dimension are identical for each slab (3rd mode) with just a varying scale (U, Fig. 1). While in many applications this decomposition has been shown to be useful (see e.g. Acar et al. 2007; Latchoumane et al. 2012), the assumption may not follow exactly the structure of many data sets. For a more general, relaxed trilinear factorization, we present a novel formulation that allows MTF to capture variation that may not be strictly trilinear. More specifically, the relaxed formulation aims to decompose the tensor $\mathcal{W}^{(t)}$ of Eq. 1 in a flexible fashion, allowing identification of trilinear structure (characteristic of CP factorization), along with structure that is specific to each slab of the tensor (bilinear, matrix structure); but also structures that are along a continuum between the two extremes. This can be seen in Fig. 3, where the patterns in $\mathcal{W}^{(2)}$ are the same for each tensor slab in MTF, but with varying scale. In contrast, rMTF allows the tensor slabs to deviate from the average patterns.

We formulate a Bayesian solution for the relaxed MTF (rMTF) problem, allowing flexible factorization. The tensors $\mathcal{X}^{t=(1: T)}$ are factorized jointly, capturing factors that are shared between all, some, or one of the views. The formulation is relaxed in the sense that the tensors $\mathcal{X}^{(t)}$ have a hierarchical decomposition that allows the model to flexibly tune between the generic matrix factorization and the trilinear $\mathrm{CP}$ factorization of the tensors. The distributional assumptions of our model are:

$$
x_{n, d, l}^{(t)} \sim \mathcal{N}\left(\mathbf{z}_{n}^{\top} \mathbf{w}_{l, d}^{(t)}, \tau_{t, l}^{-1}\right)
$$

1 http://research.cs.aalto.fi/pml/software/mtf/. 


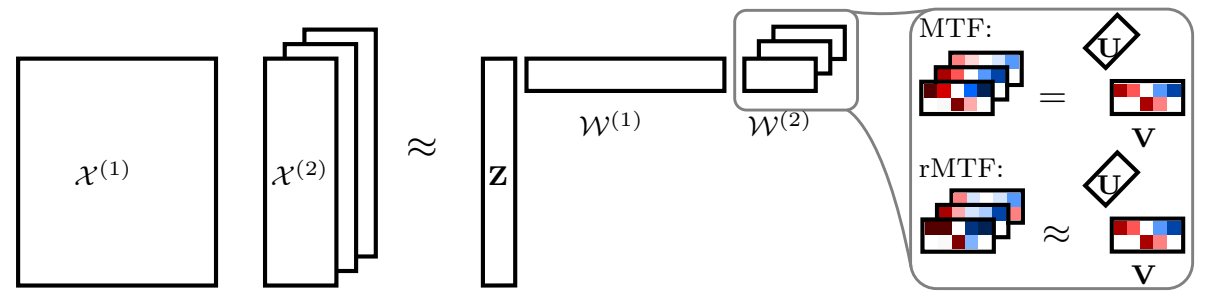

Fig. 3 An illustration of joint factorization of a matrix $\mathcal{X}^{(1)}$ and a tensor $\mathcal{X}^{(2)}$. The generation of the loading matrices corresponding to the tensor, $\mathcal{W}^{(2)}$, is shown in more detail on the right $\left(D_{2}=5\right.$ and $\left.K=2\right)$. MTF does a trilinear CP decomposition, as shown by the $\mathcal{W}^{(2)}$ slabs that equal $\mathbf{V}$ with just a scale difference. rMTF allows deviation from this, as illustrated by the slight changes in the $\mathcal{W}^{(2)}$ patterns

$$
\begin{aligned}
w_{l, d, k}^{(t)} & \sim h_{l, k}^{(t)} \mathcal{N}\left(u_{l, k}^{(t)} v_{d, k}^{(t)},\left(\alpha_{d, k}^{(t)}\right)^{-1}\right)+\left(1-h_{l, k}^{(t)}\right) \delta_{0} \\
z_{n, k}, u_{l, k}^{(t)} & \sim \mathcal{N}(0,1) \\
v_{d, k}^{(t)} & \sim \begin{cases}0, & \text { if } L_{t}=1, \\
\mathcal{N}\left(0,\left(\beta_{d, k}^{(t)}\right)^{-1}\right), & \text { otherwise. }\end{cases} \\
\alpha_{d, k}^{(t)} & \sim \begin{cases}\operatorname{Gamma}\left(a^{\alpha}, b^{\alpha}\right), & \text { if } L_{t}=1, \\
\lambda, & \text { otherwise. }\end{cases} \\
h_{l, k}^{(t)} & \sim \operatorname{Bernoulli}\left(\pi_{k}\right) \\
\pi_{k} & \sim \operatorname{Beta}\left(a^{\pi}, b^{\pi}\right) \\
\beta_{d, k}^{(t)} & \sim \operatorname{Gamma}\left(a^{\beta}, b^{\beta}\right) \\
\tau_{t, l} & \sim \operatorname{Gamma}\left(a^{\tau}, b^{\tau}\right) \\
\lambda & \sim \operatorname{Gamma}\left(a^{\lambda}, b^{\lambda}\right) .
\end{aligned}
$$

The key aspect of the model structure is the parameter $\lambda$ describing the similarity of the tensor slabs. The difference to regular MTF is illustrated in Fig. 3 (right). High $\lambda$ values indicate a factorization that is primarily driven by the low-rank CP structure and is effectively trilinear, whereas low values correspond to highly flexible bilinear multiple matrix factorization. In the tensor formulation, Tucker-1 factorization closely resembles the bilinear multiple matrix factorization, and is considered the least restrictive form of tensor factorization (Kiers 1991). By default, $\lambda$ will have a relatively uninformative prior $\left(a^{\lambda}=b^{\lambda}=1\right)$, enabling data driven inference of the tensor structure.

Alternatively, informative priors can be considered as well, if there indeed is some prior information about the structure of the tensors. Additionally, specifying $\lambda_{k}$ for each component or $\lambda_{l}$ for each tensor slab would allow learning interesting information about the data structure, namely how strongly each component or slab, respectively, is associated to the trilinear tensor structure. Similarly to MTF, the inference for rMTF is performed with Gibbs sampling, and the implementation is publicly available along with the MTF implementation.

\section{Generalized multi-tensor factorization}

The notion of coupled matrices and tensors can be formulated for all modes of all tensors, allowing decomposition of arbitrarily coupled data sets, for investigation of factors shared and 


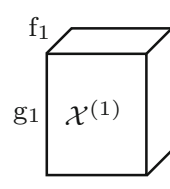

$\mathrm{g}_{2}$
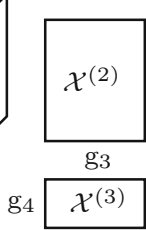
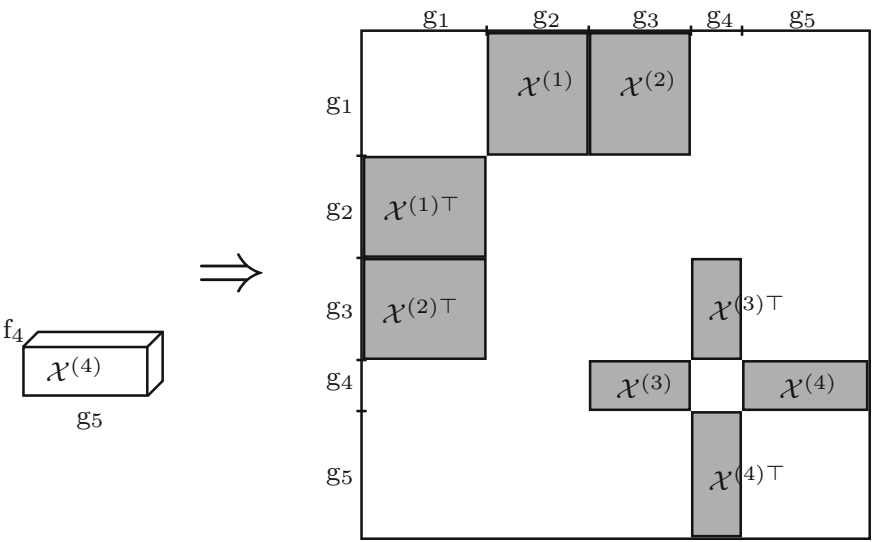

Fig. 4 Tensor collections with arbitrary pairing between the modes can be assembled into a single large sparse tensor. If the first and the second mode contain groups $\left\{\mathrm{g}_{1}, \ldots \mathrm{g}_{G}\right\}$ and the third mode groups $\left\{\mathrm{f}_{1}, \ldots \mathrm{f}_{F}\right\}$, the whole data collection forms a $\sum_{i=1}^{G}\left|\mathrm{~g}_{i}\right| \times \sum_{i=1}^{G}\left|\mathrm{~g}_{i}\right| \times \sum_{j=1}^{F}\left|\mathrm{f}_{j}\right|$ tensor. An illustration of the two first modes of this tensor is presented on the right (transpose operating on the first two modes only). A group sparse factorization for this tensor amounts to a MTF generalized to arbitrarily paired tensor collections

specific to each. To this end, we formulate the general problem of multi-tensor factorization, for data collections consisting of matrices and tensors paired in any user-defined fashion.

The joint factorization task in such multi-mode blocks can be framed as identification of a low-dimensional representation for each of the data modes. This is enabled by the observation that the distinction between samples and dimensions vanishes, as all modes of the data become analogous. Figure 4 illustrates the formulation with an example of two matrices $\mathcal{X}^{(2)} \in \mathbb{R}^{D_{1} \times D_{3} \times 1}, \mathcal{X}^{(3)} \in \mathbb{R}^{D_{4} \times D_{3} \times 1}$ and two tensors $\mathcal{X}^{(1)} \in \mathbb{R}^{D_{1} \times D_{2} \times L_{1}}, \mathcal{X}^{(4)} \in$ $\mathbb{R}^{D_{4} \times D_{5} \times L_{4}}$, paired in a non-trivial way. The task in this case is to find $K$ factors to represent each of the dimension blocks $\left(\mathrm{g}_{1}, \ldots, \mathrm{g}_{5}, \mathrm{f}_{1}, \ldots, \mathrm{f}_{4}\right)$, while capturing the common as well as distinct activity patterns that link the data sets $\mathcal{X}^{(1)}, \mathcal{X}^{(2)}, \mathcal{X}^{(3)}, \mathcal{X}^{(4)}$ together. To solve the task, we represent the entire data collection as a tensor $\widehat{\mathcal{X}} \in \mathbb{R}^{\sum D_{i} \times \sum D_{i} \times \sum L_{i}}$ (partly illustrated in Fig. 4), and the $K$ factors as the low-dimensional tensor $\mathcal{W} \in \mathbb{R}^{\sum D_{i} \times K \times \sum L_{i}}$, which has a strict block-structure, active only for regions corresponding to data sets being modeled.

Formally, for $m$ data sets collected into the tensor $\widehat{\mathcal{X}}$ the model is

$$
\hat{\mathbf{X}}_{:, ., l} \sim \mathbf{W}_{:,:, l} \mathbf{W}_{:,:, l}^{\top},
$$

where block-structure $\left\{\mathrm{g}_{1}, \mathrm{~g}_{2}, \mathrm{~g}_{3}, \mathrm{~g}_{4}, \mathrm{~g}_{5}\right\}$ is imposed by the binary variable $h_{b, k, l}$ for $k \in$ $1 \ldots K, l \in 1 \ldots L$ via a spike and slab prior. The relaxed formulation of Sect. 4 is embedded by assuming that the $\sum L_{i}$ slabs of $\mathcal{W}$ are drawn from the mean matrix $\mathbf{V} \in \mathbb{R}^{\sum D_{i} \times K}$ as

$$
\begin{aligned}
w_{d, k, l} & \sim \begin{cases}h_{b_{d}, k, l} v_{d, k}+\left(1-h_{b_{d}, k, l}\right) \delta_{0}, & \text { if } l \text { is a matrix-slab, } \\
h_{b_{d}, k, l} \mathcal{N}\left(v_{d, k} u_{l, k}, \lambda^{-1}\right)+\left(1-h_{b_{d}, k, l}\right) \delta_{0}, & \text { otherwise. }\end{cases} \\
v_{d, k} & \sim \mathcal{N}\left(0,\left(\alpha_{d, k}\right)^{-1}\right) \\
\alpha_{d, k} & \sim \operatorname{Gamma}\left(a^{\alpha}, b^{\alpha}\right),
\end{aligned}
$$

where $b_{d}$ denotes which group feature $d$ belongs to and the other priors remain unchanged. 
The binary variable $h_{b_{d}, k, l}$ learns in which group each component is active, producing the block-component activations that extend also to slabs for tensor data sets. The $\lambda$ again controls the balance between the trilinear and Tucker- 1 structure in the data. Model specification is completed by assuming normal distribution for $\widehat{\mathcal{X}}$ and a data view specific noise precision $\tau_{t}$.

The key characteristic here is that group sparsity controls the activation of each latent block-component pair instead of the data set-component pair; therefore a component's contribution in a data set can be switched off in multiple ways. For example, in matrix $\mathcal{X}^{(2)}$, the component $k$ can be switched off if either $h_{1, k, 1}=0$ or $h_{3, k, 1}=0$. For tensors, the switching notion extends to each of the $L_{t}$ slabs. This specification makes the model fully flexible and allows components with all possible sharing and specificity patterns to be learned, given enough regularization.

This formulation resembles a recent non-negative multiple tensor factorization by Takeuchi et al. (2013). We introduce a Bayesian formulation with relaxed factorization as well as segregate between shared and specific components.

\section{Related work}

The MTF problem and our solution for it are related to several matrix and tensor factorization techniques. In the following we discuss existing techniques that solve special cases of the multi-tensor factorization problem, and relate them to our work.

For a tensor coupled with one or more matrices, our MTF model can be seen as a Bayesian coupled matrix-tensor factorization (CMTF) method, which can additionally automatically infer the number and type of the components in the data, and enforce feature-level sparsity for improved regularization and interpretability. In this line of work, ours is closest to the nonprobabilistic CMTF of Acar et al. (2011, 2013a, b). They assumed an underlying CP decomposition for tensors too, and used a gradient-based least squares optimization approach. In their recent work, Acar et al. (2013a, 2014) enforced an $l_{1}$ penalty on the components assuming they can be shared or specific to data sets. However, unlike ours, they still required the data cardinality $(K)$ to be pre-specified. Determining the cardinality of tensors has been considered a challenging problem (Kolda and Bader 2009), and our method presents an intrinsic solution for this. Researchers have also used matrices as side information sources to a tensor in CMTF to show improved factorization performance (Zheng et al. 2012), while some have also studied underlying factorizations other than the $\mathrm{CP}$, such as the Tucker3 and the block-term decomposition (Narita et al. 2012; Sorber et al. 2015; Y1lmaz et al. 2011). Recently, solutions have been presented for speeding up the computation of coupled matrix tensor factorization algorithms on big data (Beutel et al. 2014; Papalexakis et al. 2014). These methods may be generalized to model multi-view matrices and tensors; however, we present a Bayesian formulation.

When all the tensors have $L_{t}=1$ and are paired in the first mode, our framework reduces to the group factor analysis (GFA) problem presented by Virtanen et al. (2012). GFA has been generalized to allow pairings between arbitrary data modes under the name collective matrix factorization (CMF) (Klami et al. 2014), which the formulation in Sect. 5 generalizes to tensors.

In tensor factorization research, a multi-view problem was recently studied under the name of multi-view tensor factorization (Khan and Kaski 2014). The goal there was to perform a joint $\mathrm{CP}$ decomposition of multiple tensors to find dependencies between data sets. This method can be seen as a special case of our model, when all data views are only tensors of the same order, paired in two modes and assuming a strict CP-type factorization. 


\section{Technical demonstration}

In this section we demonstrate the proposed MTF methods on artificial data. We compare with the multi-view matrix factorization method group factor analysis (GFA) (Virtanen et al. 2012), for which the tensors $\mathcal{X}^{(t)} \in \mathbb{R}^{N \times D_{t} \times L_{t}}$ are transformed into $L_{t}$ matrices $\mathbf{X}^{(1)}, \mathbf{X}^{(2)} \ldots \mathbf{X}^{\left(L_{t}\right)} \in \mathbb{R}^{N \times D_{t}}$, one for each slab of the tensor. In this setting, GFA corresponds to a joint matrix and Tucker-1 tensor factorization. Thus GFA presents the most flexible tensor factorization, whereas MTF does a strict CP-decomposition, and rMTF learns a representation in between these two.

\subsection{Visual example}

We start with an experiment that illustrates the properties of MTF. We generated $N=300$ data samples from MTF (Eq. 3), one matrix view with $D_{1}=50$ and one tensor view with $D_{2}=50$ and $L=30$. A total of 11 components were used to generate the data: 1 fully shared, 2 specific to the matrix and 8 to the tensor. The fully shared component was given the shape of a sine wave in the second dimension (V), as shown in Fig. 5 (left, black curves) for the first 25 features of two tensor slabs. The data set generation was repeated independently 100 times, and the component structures (that is, how many shared and specific components are inferred) detected by the models are shown in Table 1 (CP). MTF was able to find exactly one shared component in every repetition, but tended to overestimate the number of matrixspecific components (on average 3.37 vs. the true 2). GFA was very accurate in detecting the number of matrix-specific components, but overestimated the amount of other structure. Overall, MTF is able to detect the CP structure considerably more accurately than GFA, which assumes a bilinear structure in the data. The inferred posterior means of a representative data set replication for the shared component weights are shown in Fig. 5 (left). MTF detected the cardinality and component activation correctly, while GFA returned two shared components, out of which the one closer to the true parameters is shown.

To illustrate the advantages of relaxed MTF, we add some distortion to the sine wave of the shared component; the distortion is $v^{p}$, where $p=\{0.5,1.5\}$ for the first two tensor slabs, and an evenly spaced series between 0.3 and 1.7 for the rest. The weights corresponding to
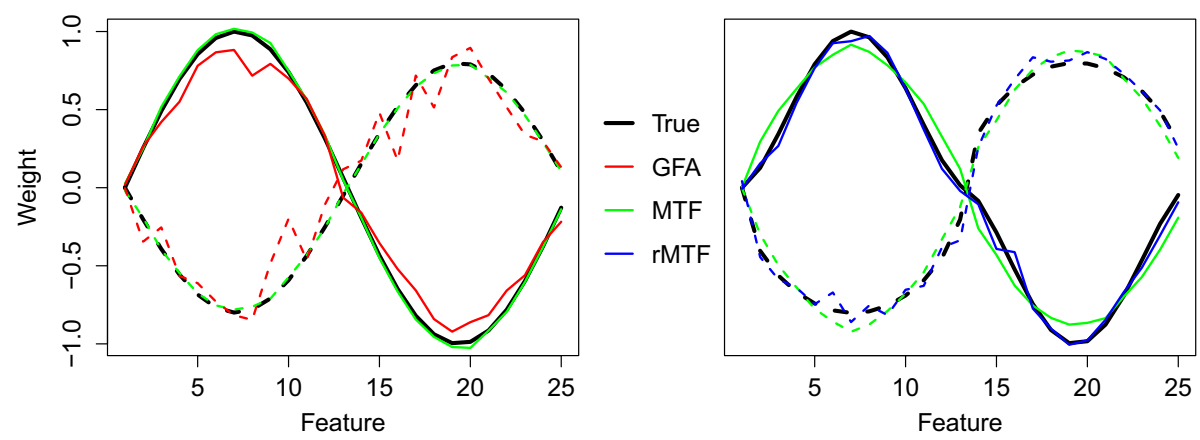

Fig. 5 Illustration of MTF inference on simulated data when the projections of the tensor are given the shape of a sine wave ("True"). The solid curve denotes the first slab and dashed the second. Left MTF infers the correct shape more accurately than multi-view matrix factorization (GFA). Right MTF cannot compensate for tensor slabs deviating from the common signal, whereas the relaxed version (rMTF) can, detecting the true parameters more accurately 
Table 1 Top: Number of (shared, matrix-specific and tensor-specific) components used to generate the data ("True") and inferred on average with different models over 100 independent simulated data sets (standard deviation in parentheses)

\begin{tabular}{|c|c|c|c|c|c|}
\hline & \multirow[t]{2}{*}{ True } & \multicolumn{2}{|l|}{$\mathrm{CP}$} & \multicolumn{2}{|l|}{ Relaxed CP } \\
\hline & & MTF & GFA & MTF & rMTF \\
\hline Shared & 1 & $1(0)$ & 2.17 (1.57) & $1.99(0.1)$ & $1.73(0.98)$ \\
\hline Matrix & 2 & $3.37(0.98)$ & $2.04(0.2)$ & $3.39(0.92)$ & $1.93(0.57)$ \\
\hline Tensor & 8 & $8.19(0.44)$ & $10.75(1.62)$ & $8.07(0.26)$ & $10.07(1.27)$ \\
\hline Correlation & 1 & $0.9999(0)$ & $0.9954(0.01)$ & $0.9999(0)$ & $0.9937(0.02)$ \\
\hline
\end{tabular}

With strict CP data generation, MTF was able to detect the correct number of components shared between the matrix and tensor in every repetition. GFA was more accurate only in detecting the number of matrix-specific components. With the shared component generated from relaxed CP, rMTF was more accurate in detecting the component structure, with the exception of overestimating the number of tensor-specific components. Bottom: The average correlations between the true and the inferred loadings of a tensor-specific component. All the models infer the structure of the specific component very accurately; MTF in particular, as the tensor-specific component has a CP form

powers 0.5 and 1.5 are shown in Fig. 5 (right, black curves). MTF cannot take into account this kind of differences in the projection weights, whereas rMTF finds a closer match to the correct weights, as shown in Fig. 5 (right). In this example, as the trilinear assumption does not hold exactly, MTF returned two shared components, out of which the one closer to the true parameters is shown. It is also worth noting that MTF nevertheless finds a more continuous estimate, as it learns the loadings for all the 30 tensor slabs jointly, instead of allowing individual variance. We additionally tested the ability of the models to detect the true component structure with 100 independent repetitions of data generation and model inference. The average number of inferred components is shown in Table 1 (Relaxed CP) for the three component types: shared, matrix-specific and tensor-specific. As the joint signal is no longer strictly generated from CP, MTF typically uses two shared components to represent it. MTF detects only the number of tensor-specific components, which are strictly CP, more accurately than rMTF. The tested methods overestimate the component numbers somewhat, producing weak spurious components in addition to the stronger true components. Overall, considering the scale of the components as well, they are fairly accurate in detecting the correct component structure in this simulation study. We also studied how accurately the components are inferred. Table 1 shows the average (absolute) correlation between a true tensor-specific component and an inferred component that resembles it the most. The Bayesian factorization methods are very accurate in this respect. Due to the $\mathrm{CP}$ generation of the tensor-specific components, this holds for MTF in particular.

\subsection{Continuum between bilinear and trilinear factorization}

In this experiment, we evaluate the performance of MTF and rMTF on the continuum between multi-view matrix factorization and matrix tensor factorization. The trilinear tensor factorization for slab $l$ of the $t$ th tensor is of the form $\sum_{k=1}^{K} u_{l, k} \mathbf{z}_{:, k} \mathbf{v}_{:, k}^{(t) \top}$, whereas the bilinear multi-view matrix factorization corresponds to $\sum_{k=1}^{K} \mathbf{z}_{:, k} \mathbf{v}_{:, k}^{(l) \top}$, where the matrix $\mathbf{V}^{(l)}$ is $a$ priori independent from all the other data views. We studied the case where neither of these assumptions is correct, but the true factorization is between the assumptions of the two mod- 


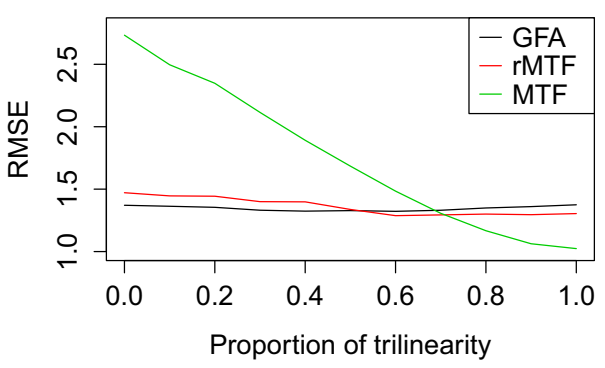

Fig. 6 A simulation study showing the prediction error (lower is better; an ideal model results in RMSE 1 and a random guess in RMSE 3) as a function of the proportion of trilinearity (vs. bilinearity) in the data; 0 corresponds to (bilinear) multi-view matrix factorization and 1 to perfectly trilinear matrix tensor factorization. As expected, MTF has poor performance when the data do not match the modeling assumptions (on the left), and top-level when they do (on the right). Matrix factorization method GFA is the ideal model when the data have close to bilinear structure, and relaxed MTF is generally close to the better one of these two, making it the most robust model. It suffers somewhat from having a more general parametrization, but is the most accurate model in the mid-region (from 0.55 to 0.7 )

els. For this, we generated $N=15$ samples of training data: one matrix with $D_{1}=50$ and one tensor with $D_{2}=50$ and $L=30$. The data set was generated with one fully shared component, 2 specific components for the matrix and 8 for the tensor. The generative model used was a weighted sum of the bilinear and trilinear factorizations. The quality of the models was evaluated by predicting 100 test data samples of one tensor slab $(l=1)$ from the rest of the tensor $(l=2, \ldots, 30)$. For this purpose, we used a two-stage approach: First the parameters were inferred from the fully observed training data, storing the ones that affect the new test samples as well (that is: $\mathbf{V}, \mathbf{U}$ and $\boldsymbol{\tau}$ ). In the second stage the latent variables $\mathbf{Z}$ and the missing parts of the test data (in this case tensor slab $l=1$ ) were sampled given $\{\mathbf{V}, \mathbf{U}, \boldsymbol{\tau}\}$. This procedure was repeated for all the training phase posterior samples and the final prediction was an average over all the predicted values.

The performance of the MTF models and bilinear GFA in this experiment can be seen in Fig. 6 (averaged over 300 repetitions); the performance was quantified with the prediction RMSE on the left-out test data set. GFA results in a seemingly constant prediction accuracy with respect to the proportion of trilinearity in the data, and is the most accurate model when the data generation process is fully bilinear, as expected. MTF assumes a strictly CPtype of decomposition, and hence varies from weak performance (bilinear data) to ideal performance (trilinear data). Relaxed MTF is the most robust approach, resulting in close to optimal prediction in most of the continuum. When the proportion of trilinearity is in the mid-region, rMTF results in the most accurate predictions. Besides the prediction accuracies, the models' abilities to detect the correct component structure were evaluated as well (data not shown). Even though the number of training samples was very low, the models were able to identify the total number of components rather accurately, but the number of shared components was generally overestimated, underestimating the number of matrix and tensor specific components. In general, prediction accuracy and component detection accuracy were in strong concordance. In the extreme case, with fully trilinear data, MTF was able to detect the exact component structure in 299 out of the 300 repetitions (with 0.9 trilinearity in 290 repetitions). GFA, on the other hand, overestimated the true component amount on average by 0.6 , and reported false weak connections between some tensor (or matrix) specific components. The low sample size $(N=15)$ suppressed the models' tendencies to report overly many (weak) components, as opposed to the simulation study in Sect. 7.1. 


\section{Applications}

In this section we demonstrate the use of the proposed MTF methods in two applications: functional neuroimaging and structural toxicogenomics. To illustrate the strengths of the new methods, we compare them with tensor factorization methods that are the most closely related to them. In particular, we compare with coupled matrix tensor factorization (Acar et al. 2013b), which decomposes a tensor along with a coupled matrix as side information. The available implementation ${ }^{2}$ uses $\mathrm{CP}$ as the underlying factorization for the tensor, as does our MTF. Additionally, we compare against an asymmetric version of coupled matrix tensor factorization (ACMTF) (Acar et al. 2013a, 2014), which allows both private and shared components in the data collection. CMTF and ACMTF are the closest existing tensor baselines and are non-probabilistic formulations. We also compare our method to a multiview matrix factorization method group factor analysis (GFA) (Virtanen et al. 2012) by transforming the tensors $\mathcal{X}^{(t)} \in \mathbb{R}^{N \times D_{t} \times L_{t}}$ into $L_{t}$ matrices $\mathbf{X}^{(1)}, \mathbf{X}^{(2)} \ldots \mathbf{X}^{\left(L_{t}\right)} \in \mathbb{R}^{N \times D_{t}}$, one for each slab of the tensor, as this corresponds to a joint matrix and Tucker-1 tensor factorization.

Model complexity was determined in a data-driven way, by setting $K$ large enough so that some of the inferred components became shut down. The model parameters $a^{\pi}, b^{\pi}, a^{\lambda}, b^{\lambda}$ were initialized to 1 to represent uninformative symmetric priors. Feature-level sparsity was assumed with parameters $a^{\alpha}, b^{\alpha}$ set to $10^{-3}$, while high noise in the data was accounted for by initializing the noise hyperparameters $a^{\tau}, b^{\tau}$ for a signal-to-noise ratio (SNR) of 1 . All the remaining model parameters were learned. CMTF and ACMTF were run with $K$ values inferred from MTF, as they are unable to learn $K$. ACMTF was run with sparsity setting of $10^{-3}$, as recommended by the authors (Acar et al. 2013a). For all the models, the predictions for missing data were averaged over 7 independent sampling chains/runs to obtain robust findings. For missing value predictions, we used the two-stage out-of-sample prediction scheme discussed in the previous section. The data were centered to avoid using components to model the feature means, and unit-normalized to give equivalent importance for each feature.

\subsection{Functional neuroimaging}

A key task in many neuroimaging studies is to find the response related to a stimulus. This is an interesting problem in natural stimulation and multi-subject settings in particular. MTF can be applied in this scenario directly, as the stimulus can generally be represented with a matrix of $N$ samples (time points) and $D_{1}$ features, whereas the imaging measurements are a tensor with $N$ samples, dimension $D_{2}$ (e.g. MEG channels) and depth $L$ (subjects). We analyzed a data set presented by Koskinen and Seppä (2014), where $L=9$ subjects (one out of ten omitted due to unsuccessful recordings) listened to an auditory book for approximately $60 \mathrm{~min}$, while being measured in a magnetoencephalography (MEG) device. In this context, analysis with multi-matrix factorization methods (with subjects regarded as different data views) would assume that the subjects a priori do not have any shared information. MTF, on the other hand, aims to decompose the data such that the latent time series (components) have equal feature weights for all the subjects, just scaled differently. Although the imaging device is the same for all the subjects, they will share neither the exactly same brain structure nor functional responses. This makes rMTF a promising model for neuroimaging applications.

The data set was preprocessed in a similar fashion as in Koskinen and Seppä (2014). Namely, the $60 \mathrm{~min}$ of MEG recordings were wavelet-transformed with central frequency

2 http://www.models.life.ku.dk/ acare/CMTF_Toolbox. 

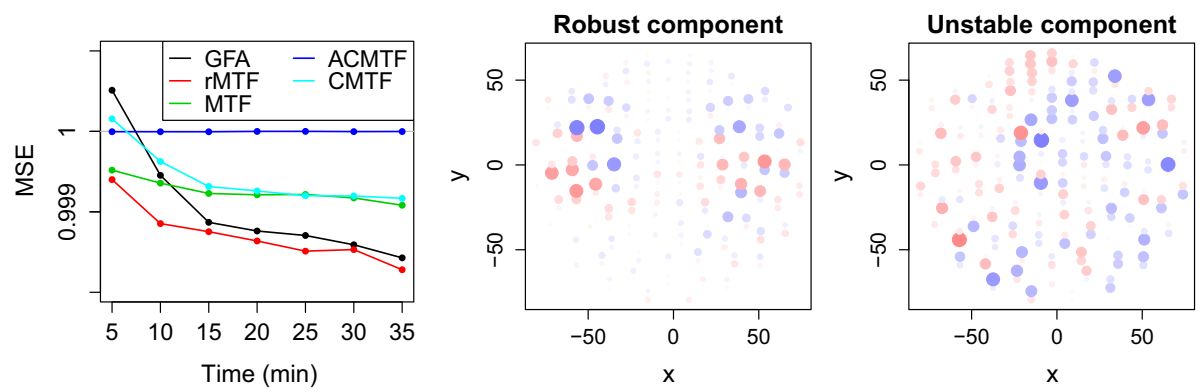

Fig. 7 Left The mean squared errors (MSE) on predicting (later) brain responses to stimuli as a function of (earlier) training data size (shown as experimental time; ranging from 2500 to 17,500 samples). Predicting mean of training data results in MSE of 1. Middle The feature weights, averaged over subjects, of a robust rMTF component shown in the MEG channel space (top view). Activations are focused around the auditory areas. Right The averaged feature weights of an unstable GFA component shown in the MEG channel space

0.54 , decreasing the sample size to $N=28547$. The recordings were preprocessed with the signal-space-separation (SSS) method (Taulu et al. 2004) and furthermore with PCA (jointly for all the subjects) to reduce the dimensionality from 204 (MEG channels) to the number of degrees of freedom left after the SSS procedure $\left(D_{2}=70\right)$. As there is a delay in brain responses corresponding to the stimulus, the mel-frequency cepstrum coefficients (MFCC, computed with the Matlab toolbox voicebox) describing the power spectrum of the auditory stimulus $\left(D_{1}=13\right)$ were shifted to have maximal correlation with the response, and then downsampled and wavelet-transformed to match the MEG recordings.

We inferred the matrix-tensor decompositions with the two methods introduced in this paper and the three comparison methods. The decomposition was inferred from the $n$ first measurements, and the models were then used to predict all the later MEG measurements given the later audio. Relaxed MTF and GFA were run with $K=500$, leaving empty components with every training sample size (final component amount ranging from 132 to 380 , depending on the sample size). For MTF, even $K=700$ (larger than the total data dimensionality) was not sufficient, suggesting the data do not fully fit the strong $\mathrm{CP}$ assumptions, and extra components have to be used to explain away some non-CP variation (structured noise). No degeneracy was observed, however, and we used a stronger regularizing prior for MTF ( $a^{\pi}=\frac{1}{b^{\pi}}=10^{-3}$ and peaked noise prior for SNR of 1 ), ending up with around 500 active components. Due to memory requirements, ACMTF was run with only $K=70$, using over $10 \mathrm{~GB}$ of RAM. The Bayesian methods inferred with Gibbs sampler were run with 3000 burn-in samples, returning 40 posterior samples with 10 sample thinning for each sampler chain. We evaluated the convergence of the methods based on the reconstruction of the training data in the posterior samples. Applying the Geweke diagnostic (Geweke 1992) under this framework showed that all the chains were converged. The single chain running times of the Bayesian models initialized with $K=500$ were approximately $17 \mathrm{~h}$ with the largest training data (4h with $5 \mathrm{~min}$ of training data). For MTF, initialized with $K=700$, the corresponding running times were roughly 70 and $24 \mathrm{~h}$.

Relaxed MTF learned the stimulus-response relationship most accurately for a wide range of training data sizes (Fig. 7), whereas the trilinear factorization of MTF seems to be too strict and the multi-matrix factorization of GFA too flexible. Despite the challenges in the model complexity determination for MTF, likely due to the overly strict modeling assumptions, it still was able to infer a meaningful factorization. CMTF showed similar performance as MTF once it was given enough training samples. The sparse solution of ACMTF did 
not deviate from null prediction even though different sparsity parameters and convergence criteria were tested. Relaxed MTF was significantly superior to all the other methods with all the training set sizes ( $p<0.05$; pairwise t-test for each model pair, with MSEs of individual predicted time points as the samples). As the MSEs are close to one and their numerical differences are small, it is worth emphasizing that, especially in natural stimulus settings, the SNR of MEG experiments is very low (Koskinen and Seppä 2014). For practical use on data sets with challengingly few samples it is an important finding that rMTF learned as accurate a stimulus-response relationship as GFA with roughly half of the training set size on this data set. As overly long neuroimaging experiments tend to cause decreased signal-to-noise ratio (Hansen et al. 2010), relaxed MTF may offer significant benefit in this area.

The most robust finding of the factorization models in this application is the brain response to the energy of the speech signal. Of the 13 acoustic MFCC features, 9 are highly dependent on the signal energy and hence two-peaked, corresponding to words and breaks between the words. A robust component found in all the rMTF chains had similar two-peaked structure, and was found to be active in the auditory areas of the brain (Fig. 7, middle). With enough samples, GFA was able to detect this component robustly as well, but it produced more unstable components present in individual sampling chains only. These components had no clear structure in the MEG channels, as shown in Fig. 7, and are hence likely to be artifacts explaining noise in the recordings. No other robust shared components were found with rMTF, likely because we analyzed the relationship between the stimulus and the brain response at only one time lag. Various brain responses occur at different lags; in this experiment we focused on the initial response, simple auditory processing of the heard sound. For a more thorough neuroscientific analysis it would be important to take the temporal nature of the events more directly into account. Besides the typically 1-4 fully shared components (one robust over most of the chains), rMTF and GFA typically had 1-4 components specific to the acoustic features and around 200 describing the MEG measurements, either active for all the subjects, or for a subset of them. The wide range of MEG-specific components describe brain activity unrelated to the task, or not sufficiently described by the acoustic features. From the experimental perspective of finding stimulus-related activity, these components can be thought to describe structured noise.

\subsection{Structural toxicogenomics}

We next analyzed a novel drug toxicity response problem, where the tensors arise naturally when gene expression responses of multiple drugs are measured for multiple diseases (different cancers) across the genes. The data contain three views, the structural descriptors of drugs (a matrix), measurement of post-treatment gene expression (a tensor), and drug toxicity (a tensor) as shown in Fig. 8. As drugs have several diverse effects on cells, they can be hypothesized to have been generated by underlying factors, some of which may be common across all diseases while others specific to only few.

In this setting, MTF can be used to answer two key questions: (1) which parts of the responses are specific to individual types of cancer and which occur across cancers, and which of these responses are related to known structural properties of the drugs; and (2) can we use the links from gene expression responses along with structural properties of drugs, to predict toxicity of an unseen drug.

For the first problem, the response patterns of drugs, if uncovered, can help understand the mechanisms of toxicity (Hartung et al. 2012). The identification of links from structural properties of drugs (a matrix) to the gene/toxic responses (tensors) opens up the opportunity 

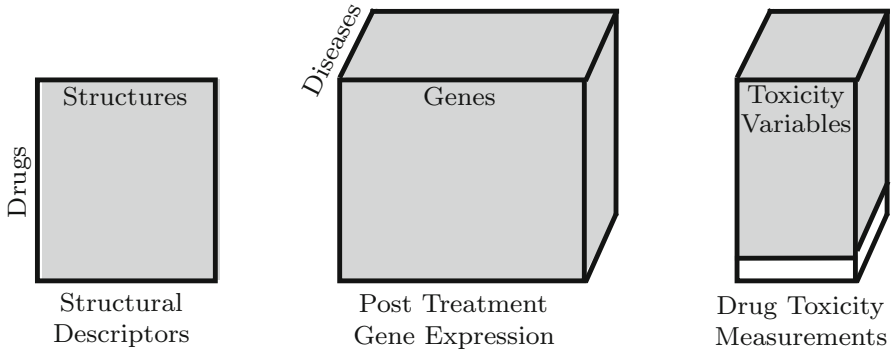

Fig. 8 Structural toxicogenomics data set with a matrix of drug descriptors paired with a tensor of gene expression responses of different diseases to the drugs, and the corresponding toxicity profiles

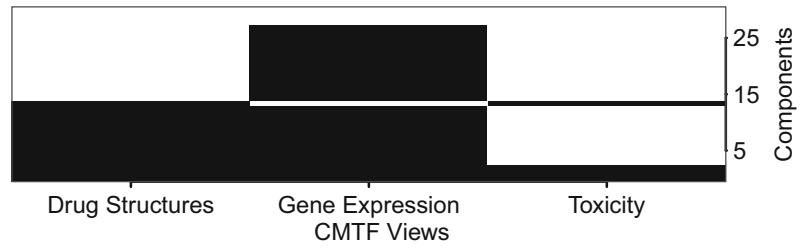

Fig. 9 Component activity plot for the structural-toxicogenomics data set (black active, white: all zeros). The model found 3 interesting components active in all the views, capturing patterns shared by structures of drugs, their disease-specific gene expression responses, and the toxicity measurements. These components capture the toxic responses of drugs

for drug-designers to better understand the functional effects of drugs' structural fragments (Khan et al. 2014). Secondly, it is interesting to explore how well our method predicts unseen responses by using multiple side-information sources and the structure in the data.

The data set contained three views (Fig. 8). The first contained structural descriptors of $N=73$ drugs. The descriptors known as functional connectivity fingerprints FCFP4 represent the presence or absence of a structural fragment in each compound. For this data set the drugs are described by $D_{1}=290$ small fragments, forming a matrix of 73 drugs by 290 fragments. The second view contains the post-treatment differential gene expression responses $D_{2}=1106$ of $N=73$ drugs, as measured over multiple diseases or here cancer types, $L=3$. The third view contained the corresponding drug sensitivity measurements, $D_{3}=3$. The two tensors are paired with the common identity of $N=73$ drugs and $L=3$ cancer types, while the drug structure matrix is paired with the tensors on the common set of $N=73$ drugs. The gene expression data were obtained from the ConnectivityMap (Lamb 2006) that contained response measurements of three different cancers: Blood Cancer, Breast Cancer and Prostate Cancer. The data were processed so that gene expression values represent up (positive) or down (negative) regulation from the untreated (base) level. Strongly regulated genes were selected, resulting in $D_{2}=1106$. The Structural descriptors (FCFP4) of the drugs were computed using the Pipeline Pilot software ${ }^{3}$ by Accelrys. The drug screen data for the three cancer types were obtained from the NCI-60 database (Shoemaker 2006), measuring toxic effects of drug treatments via three different criteria: GI50 (50\% growth inhibition), LC50 (50\% lethal concentration) and TGI (total growth inhibition). The data were processed to represent the drug concentration used in the connectivity map to be positive (when toxic) and negative indicating non-toxic. The methods were run with 5000 burn-in

\footnotetext{
3 http://accelrys.com/products/pipeline-pilot/.
} 


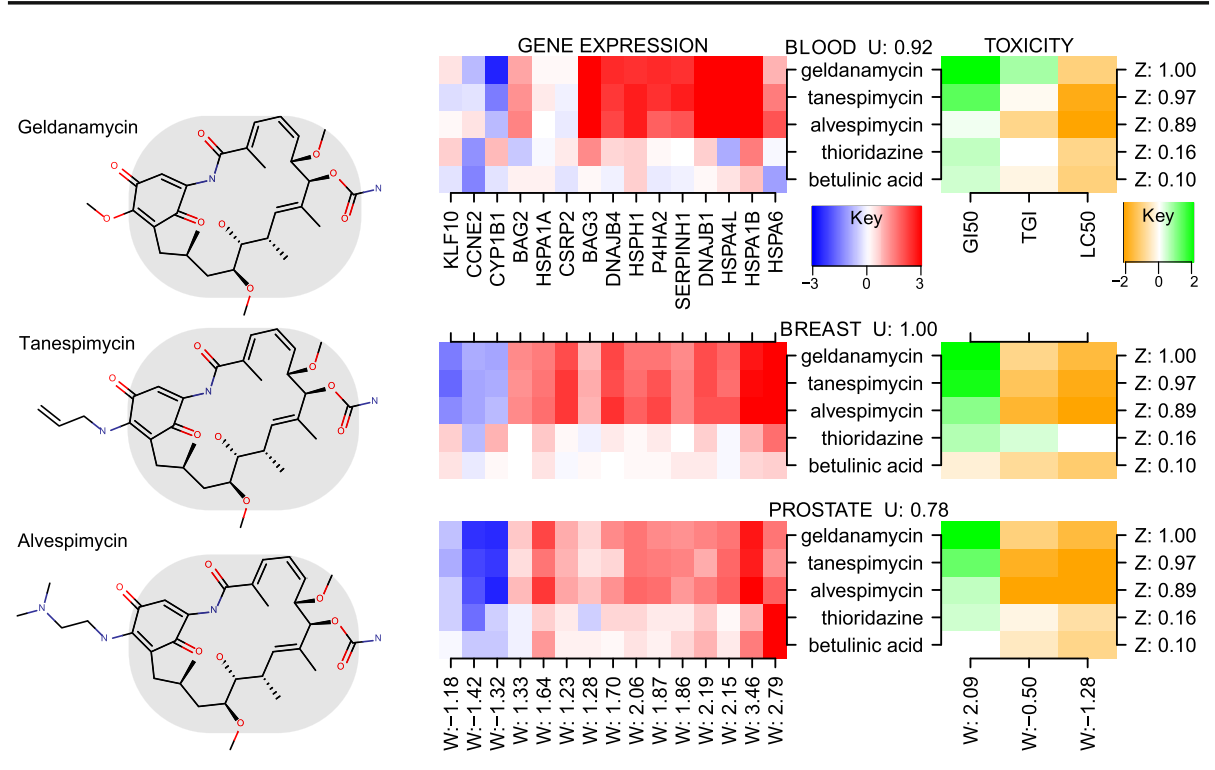

Fig. 10 An illustrative example: Component 1 captures the well-known heatshock protein response. The model identifies the common structural descriptors of the drugs (left) as drivers of the biological (middle) and toxic (right) responses. The responses are shown for the top genes and all toxicity variables, and top drugs for all the three cell lines (rows), along with the $\mathbf{Z}, \mathbf{U}$ and $\mathbf{W}$ loadings (shown at the ticks). The top genes and drugs are identified as those with the highest loadings in $\mathbf{W}$ and $\mathbf{Z}$ respectively. This component links structures of the heatshock protein inhibitor drugs (circled with gray on the left) with strong upregulation of the heatshock protein genes (red) to high toxicity (green) (Color figure online)

samples, returning 40 posterior samples with 10 sample thinning for each sampler chain. Convergence was examined as in Sect. 8.1, and all except $\sim 25 \%$ of the rMTF chains had converged. The single chain running times of the Bayesian models initialized with $K=30$ were around $1 \mathrm{~h}$.

MTF resulted in 3 components shared between the structural descriptors, the gene expression and toxicity views, revealing that some patterns are indeed shared (Fig. 9). Model complexity was again selected, as in the previous section, by assigning $K$ large enough, here $K=30$, such that then the sparsity prior shuts some of them off (to zeros). The 3 shared components can be used to form hypotheses about underlying biological processes that characterize toxic responses of drugs, and we find all three of them to be well linked to either established biology or potentially novel findings.

The first component captures the well-known heatshock protein response, of the three HSP90 inhibitor drugs (Fig. 10, left). The response is characterized by a strong upregulation of heatshock genes in all three cancers (Fig. 10, middle) and the corresponding high toxicity indications in GI50 (Fig. 10, right). The component identifies similarities between the three close structurally analogous drugs, which is in line with knowledge that the drugs directly bind to the HSP90 protein (Stebbins et al. 1997). The heatshock protein inhibition response has already been well studied for treatment of cancers (Kamal 2003), evaluating its potential therapeutic efficacy. This trilinear MTF component could have been important in revealing the response, had the mechanism not already been discovered.

The second component captures DNA damage response of several structurally similar cardiac glycoside drugs and a structurally different drug, bisacodyl, which is a laxative. Interestingly, our component found the response of bisacodyl to be specific to only one of 
Table 2 Structural toxicogenomics: toxicity prediction of an unseen drug given its structural descriptors and genomic responses.

\begin{tabular}{llllll}
\hline & MTF & rMTF & CMTF & ACMTF & GFA \\
\hline Mean & 0.579 & 0.584 & 0.692 & 0.727 & 0.642 \\
StdError & 0.062 & 0.064 & 0.079 & 0.088 & 0.070
\end{tabular}

Average Prediction RMSE is given over the entire set of drugs, with lower values signifying better performance. A paired t-test over the prediction RMSE of these drugs was carried out to test the significance of the difference between the reported methods. MTF outperforms GFA, CMTF and ACMTF significantly with $t$ test $p$ values $<0.02$

the cancer types. The link of bisacodyl with cardiac glycosides has very recently been found (Iorio et al. 2010), but the possible cancer specificity, which comes out naturally with our approach, is new.

The third and final shared component captures a common response of protein synthesis inhibitors along with an anti-metabolite (8-azaguanine) drug. Interestingly, the response is specific to two of the three cancer types, namely blood and prostate cancers. With 8azaguanine having been used in blood cancer before (Colsky et al. 1955), our component opens up an interesting opportunity for its exploration in prostate cancer.

For completeness, we also examined the first view-specific component, which captured a non-toxic response of several sequence-specific DNA binding transcription factor genes. The response is driven by two drugs, nystatin and primaquine. Nystatin is an anti-fungal drug while primaquine (an anti-malarial drug) is already well-established for use in the treatment of fungal pneumonia (Noskin et al. 1992).

This application demonstrated the model's ability to identify both established and novel links between multiple views. Systematic studies along these lines could be very valuable in precision medicine for targeting specific disease types, and in drug design when tailoring drugs to match a desired response profile.

We next evaluated the model's ability to predict the toxicity response of a new drug, by using the gene expression data (tensor) and the structural descriptors (matrix). Both are used as side information sources, coupled in a multi-view setting. This is done by modeling the dependencies between all the observed data sets and then using the learned dependencies to predict the toxicity response of a new drug, given the side information sources. The entire toxicity slab (shaded white in Fig. 8) for each drug is predicted using its gene expression and structural descriptors. We compared with the existing methods GFA, CMTF and ACMTF as baselines. GFA was run by transforming the gene expression and toxicity tensors into matrices, one for each of the $L=3$ slabs. We performed leave-one-out prediction and report the average prediction error of unseen drugs (RMSE) in Table 2. The results demonstrate that MTF predicts drug toxicity of unseen drugs significantly better, confirming that it solves well the task for which it was designed.

\section{Discussion}

We introduced Bayesian multi-tensor factorization (MTF) and as its special case the first Bayesian formulation of joint matrix-tensor factorization, extending the former formulation further to multiple sets of paired matrices and tensors. Our model decomposes the data views into factors that are shared between all or some of the views, and those that are specific 
to each. It also learns the total number and type of the factors automatically for each data collection.

We simultaneously extended our novel formulation to explore a relaxed underlying tensor factorization problem, automatically moving between the CP-type of a trilinear model and a generalized variant of a Tucker- 1 type of decomposition. The CP and the Tucker-1 decompositions fall out as special cases of the relaxed variant. This is important as Tucker-1, in particular, is suitable when data sets have minimal trilinear structure, while the CP-type trilinear decomposition in this paper has the advantage of being interpretable analogously to the matrix factorizations more familiar to most analysts.

We validated the models' performances in identifying the correct components on simulated data, and illustrated that the relaxed factorization performs well when the structure of the data is unknown or not strictly CP or Tucker-1 type. The models' performances were then demonstrated on a new structural toxicogenomics problem and on stimulus-response relationship analysis in a neuroimaging experiment, yielding interpretable findings matching with some expected effects and recent discoveries, and including also potential new innovations. The experiments indicated that taking the appropriate structure of the data into account makes the results both more accurate and easily interpretable.

Our work opens up the opportunity for novel applications and integrative studies of diverse and partially coupled data views, both for predictive purposes and feature identification.

Acknowledgments This work was financially supported by the Academy of Finland (Finnish Centre of Excellence in Computational Inference Research COIN, Grant No. 251170; and ChemBio project, Grant No. 140057) and the Finnish Graduate School in Computational Sciences (FICS). We would like to thank Miika Koskinen for his help with the neuroscience application, and Juuso Parkkinen for providing the toxicity profiles. The calculations presented above were performed using computer resources within the Aalto University School of Science "Science-IT" project.

\section{Compliance with ethical standards}

Conflict of interest The authors declare that they have no conflicts of interest.

\section{References}

Acar, E., Aykut-Bingol, C., Bingol, H., Bro, R., \& Yener, B. (2007). Multiway analysis of epilepsy tensors. Bioinformatics, 23(13), 10-18.

Acar, E., Kolda, T. G., Dunlavy, D. M. (2011). All-at-once optimization for coupled matrix and tensor factorizations. arXiv:1105.3422.

Acar, E., Lawaetz, A. J., Rasmussen, M. A., \& Bro, R. (2013a). Structure-revealing data fusion model with applications in metabolomics. In 35th annual international conference of the IEEE on engineering in medicine and biology society (EMBC) (pp. 6023-6026).

Acar, E., Rasmussen, M. A., Savorani, F., Naes, T., \& Bro, R. (2013b). Understanding data fusion within the framework of coupled matrix and tensor factorizations. Chemometrics and Intelligent Laboratory Systems, 129, 53-63.

Acar, E., Papalexakis, E., Grdeniz, G., Rasmussen, M., Lawaetz, A., Nilsson, M., et al. (2014). Structurerevealing data fusion. BMC Bioinformatics, 15(1), 239.

Bach, F. R., \& Jordan, M. I. (2005). A probabilistic interpretation of canonical correlation analysis. Tech. Rep. 688 , Department of Statistics, University of California, Berkeley.

Beutel, A., Kumar, A., Papalexakis, E. E., Talukdar, P. P., Faloutsos, C., \& Xing, E. P. (2014). Flexifact: Scalable flexible factorization of coupled tensors on hadoop. In M. Zaki, Z. Obradovic, P. N. Tan, A. Banerjee, C. Kamath, S. Parthasarathy (Eds.) SIAM international conference on data mining (pp. 109-117).

Carroll, J. D., \& Chang, J. J. (1970). Analysis of individual differences in multidimensional scaling via an n-way generalization of Eckart-Young decomposition. Psychometrika, 35(3), 283-319. 
Cattell, R. B. (1944). Parallel proportional profiles and other principles for determining the choice of factors by rotation. Psychometrika, 9(4), 267-283.

Colsky, J., Meiselas, L. E., Rosen, S. J., \& Schulman, I. (1955). Response of patients with leukemia to 8azaguanine. Blood, 10(5), 482-492.

Geweke, J. (1992). Evaluating the accuracy of sampling-based approaches to the calculation of posterior moments. In J. M. Bernardo, J. O. Berger, A. P. Dawid, \& A. F. M. Smith (Eds.), Bayesian statistics (Vol. 4, pp. 169-193). New York: Oxford University Press.

Hansen, P., Kringelbach, M., \& Salmelin, R. (2010). MEG: An introduction to methods. New York: SpringerVerlag.

Hardoon, D. R., Szedmak, S., \& Shawe-Taylor, J. (2004). Canonical correlation analysis: An overview with application to learning methods. Neural Computation, 16(12), 2639-2664.

Harshman, R. A. (1970). Foundations of the PARAFAC procedure: Models and conditions for an explanatory multimodal factor analysis. UCLA Working Papers in Phonetics, 16, 1-84.

Harshman, R. A., \& Lundy, M. E. (1994). PARAFAC: Parallel factor analysis. Computational Statistics \& Data Analysis, 18(1), 39-72.

Hartung, T., Vliet, E. V., Jaworska, J., Bonilla, L., Skinner, N., \& Thomas, R. (2012). Food for thoughtSystems toxicology. ALTEX Alternatives to Animal Experimentation, 29(2), 119-128.

Hotelling, H. (1936). Relations between two sets of variates. Biometrika, 28(3), 321-377.

Iorio, F., Bosotti, R., Scacheri, E., Belcastro, V., Mithbaokar, P., Ferriero, R., et al. (2010). Discovery of drug mode of action and drug repositioning from transcriptional responses. Proceedings of the National Academy of Sciences, 107(33), 14,621-14,626.

Kamal, A., et al. (2003). A high-affinity conformation of Hsp90 confers tumour selectivity on Hsp90 inhibitors. Nature, 425(6956), 407-410.

Khan, S. A., \& Kaski, S. (2014). Bayesian multi-view tensor factorization. In T. Calders, F. Esposito, E. Hüllermeier, \& R. Meo (Eds.), Machine learning and knowledge discovery in databases, ECML PKDD 2014 (pp. 656-671). berlin: Springer.

Khan, S. A., Virtanen, S., Kallioniemi, O. P., Wennerberg, K., Poso, A., \& Kaski, S. (2014). Identification of structural features in chemicals associated with cancer drug response: A systematic data-driven analysis. Bioinformatics, 30(17), i497-i504.

Kiers, H. A. (1991). Hierarchical relations among three-way methods. Psychometrika, 56(3), 449-470.

Klami, A., Virtanen, S., \& Kaski, S. (2013). Bayesian canonical correlation analysis. Journal of Machine Learning Research, 14, 965-1003.

Klami, A., Bouchard, G., \& Tripathi, A. (2014). Group-sparse embeddings in collective matrix factorization. In International conference on learning representations.

Klami, A., Virtanen, S., Leppäaho, E., \& Kaski, S. (2015). Group factor analysis. IEEE Transactions on Neural Networks and Learning Systems, 26(9), 2136-2147.

Kolda, T., \& Bader, B. (2009). Tensor decompositions and applications. SIAM Review, 51(3), 455-500.

Koskinen, M., \& Seppä, M. (2014). Uncovering cortical MEG responses to listened audiobook stories. Neurolmage, 100, 263-270.

Kruskal, J. B. (1977). Three-way arrays: Rank and uniqueness of trilinear decompositions, with application to arithmetic complexity and statistics. Linear Algebra and its Applications, 18(2), 95-138.

Lamb, J., et al. (2006). The connectivity map: Using gene-expression signatures to connect small molecules, genes, and disease. Science, 313(5795), 1929-1935.

Latchoumane, C. F. V., Vialatte, F. B., Solé-Casals, J., Maurice, M., Wimalaratna, S. R., Hudson, N., et al. (2012). Multiway array decomposition analysis of EEGs in alzheimer's disease. Journal of Neuroscience Methods, 207(1), 41-50.

Mitchell, T. J., \& Beauchamp, J. J. (1988). Bayesian variable selection in linear regression. Journal of the American Statistical Association, 83(404), 1023-1032.

Narita, A., Hayashi, K., Tomioka, R., \& Kashima, H. (2012). Tensor factorization using auxiliary information. Data Mining and Knowledge Discovery, 25(2), 298-324.

Neal, R. M. (1996). Bayesian learning for neural networks. New York: Springer-Verlag.

Noskin, G. A., Murphy, R. L., Black, J. R., \& Phairn, J. P. (1992). Salvage therapy with clindamycin/primaquine for pneumocystis carinii pneumonia. Clinical Infectious Diseases, 14(1), 183-188.

Papalexakis, E. E., Faloutsos, C., Mitchell, T., Talukdar, P. P., Sidiropoulos, N. D., \& Murphy, B. (2014). TurboSMT: Accelerating coupled sparse matrix-tensor factorizations by 200x. In M. Zaki, Z. Obradovic, P. N. Tan, A. Banerjee, C. Kamath, S. Parthasarathy (Eds.), SIAM international conference on data mining (pp. 118-126).

Shoemaker, R. H. (2006). The NCI60 human tumour cell line anticancer drug screen. Nature Reviews Cancer, $6(10), 813-823$. 
Smilde, A. K., Westerhuis, J. A., \& Boque, R. (2000). Multiway multiblock component and covariates regression models. Journal of Chemometrics, 14(3), 301-331.

Sorber, L., Van Barel, M., \& De Lathauwer, L. (2015). Structured data fusion. IEEE Journal of Selected Topics in Signal Processing, 9(4), 586-600.

Sørensen, M., \& De Lathauwer, L. D. (2015). Coupled canonical polyadic decompositions and (coupled) decompositions in multilinear rank- $\left(L_{r, n}, L_{r, n}, 1\right)$ terms-Part i: Uniqueness. SIAM Journal on Matrix Analysis and Applications, 36(2), 496-522.

Stebbins, C. E., Russo, A. A., Schneider, C., Rosen, N., Hartl, F. U., \& Pavletich, N. P. (1997). Crystal structure of an Hsp90-geldanamycin complex: Targeting of a protein chaperone by an antitumor agent. Cell, 89(2), 239-250.

Takeuchi, K., Tomioka, R., Ishiguro, K., Kimura, A., \& Sawada, H. (2013). Non-negative multiple tensor factorization. In: 2013 IEEE 13th international conference on data mining (ICDM) (pp. 1199-1204). doi:10.1109/ICDM.2013.83.

Taulu, S., Kajola, M., \& Simola, J. (2004). Suppression of interference and artifacts by the signal space separation method. Brain Topography, 16(4), 269-275.

Tucker, L. R. (1966). Some mathematical notes on three-mode factor analysis. Psychometrika, 31(3), $279-311$.

Virtanen, S., Klami, A., Khan, S. A., \& Kaski, S. (2012) Bayesian group factor analysis. In N. Lawrence, M. Girolami (Eds.), Proceedings of the fifteenth international conference on artificial intelligence and statistics (pp. 1269-1277).

Yılmaz, K. Y., Cemgil, A. T., \& Simsekli, U. (2011). Generalised coupled tensor factorisation. In J. ShaweTaylor, R. S. Zemel, P. L. Bartlett, F. Pereira, \& K. Q. Weinberger (Eds.), Advances in neural information processing systems (Vol. 24, pp. 2151-2159).

Zheng, V. W., Zheng, Y., Xie, X., \& Yang, Q. (2012). Towards mobile intelligence: Learning from GPS history data for collaborative recommendation. Artificial Intelligence, 184, 17-37. 